\title{
Endoscopic Ultrasound-Guided Gallbladder Drainage Using a Lumen-Apposing Metal Stent for Acute Cholecystitis: A Systematic Review
}

\author{
Deepanshu Jain ${ }^{1}$, Bharat Singh Bhandari ${ }^{2}$, Nikhil Agrawal ${ }^{3}$ and Shashideep Singhal ${ }^{4}$ \\ ${ }^{1}$ Division of Gastroenterology and Hepatology, Department of Digestive Diseases and Transplantation, Internal Medicine, Einstein Healthcare \\ Network, Philadelphia, PA, ${ }^{2}$ Department of Internal Medicine, Saint Vincent Hospital, Worcester, MA, ${ }^{3}$ Department of Internal Medicine, University \\ of Buffalo, Buffalo, NY, ${ }^{4}$ Division of Gastroenterology, Hepatology and Nutrition, University of Texas Health Science Center at Houston, Houston, \\ TX, USA
}

Surgery remains the standard treatment for acute cholecystitis except in high-risk candidates where percutaneous transhepatic gallbladder drainage (PT-GBD), endoscopic transpapillary cystic duct stenting (ET-CDS), and endoscopic ultrasound-guided gallbladder drainage (EUS-GBD) are potential choices. PT-GBD is contraindicated in patients with coagulopathy or ascites and is not preferred by patients owing to aesthetic reasons. ET-CDS is successful only if the cystic duct can be visualized and cannulated. For 189 patients who underwent EUS-GBD via insertion of a lumen-apposing metal stent (LAMS), the composite technical success rate was $95.2 \%$, which increased to $96.8 \%$ when LAMS was combined with co-axial self-expandable metal stent (SEMS). The composite clinical success rate was 96.7\%. We observed a small risk of recurrent cholecystitis (5.1\%), gastrointestinal bleeding (2.6\%) and stent migration (1.1\%). Cautery enhanced LAMS significantly decreases the stent deployment time compared to non-cautery enhanced LAMS. Prophylactic placement of a pigtail stent or SEMS through the LAMS avoids re-interventions, particularly in patients, where it is intended to remain in situ indefinitely. Limited evidence suggests that the efficacy of EUS-GBD via LAMS is comparable to that of PT-GBD with the former showing better results in postoperative pain, length of hospitalization, and need for antibiotics. EUS-GBD via LAMS is a safe and efficacious option when performed by experts. Clin Endosc 2018;51:450-462

Key Words: Acute cholecystitis; Endoscopic ultrasound; Lumen-apposing metal stent

\section{INTRODUCTION}

Approximately 20 million Americans are estimated to have gallstones, and approximately 300,000 cholecystectomies are performed annually. ${ }^{1}$ Approximately $1 \%-2 \%$ of patients with asymptomatic gallstones become symptomatic each year. Acute

Received: January 7, 2018 Revised: February 14, 2018

Accepted: February 18, 2018

Correspondence: Shashideep Singhal

Division of Gastroenterology, Hepatology and Nutrition, University of Texas Health Science Center at Houston, 6431 Fannin, MSB 4.234, Houston, TX 77030, USA

Tel: +1-713-500-6677, Fax: +1-713-500-6699, E-mail: sdsinghal@gmail.com ORCID: https://orcid.org/0000-0002-8783-4889

(c) This is an Open Access article distributed under the terms of the Creative Commons Attribution Non-Commercial License (http://creativecommons.org/ licenses/by-nc/3.0) which permits unrestricted non-commercial use, distribution, and reproduction in any medium, provided the original work is properly cited. cholecystitis, which is the most common complication of gallstones occurs in $10 \%$ of symptomatic patients. ${ }^{2}$

Surgery-open or laparoscopic (performed preferentially) is the standard treatment for acute cholecystitis. ${ }^{3}$ Cholecystectomy rates were observed to increase rapidly initially, after which they stabilized in the late 1990s and currently may even be declining in the U.S. ${ }^{4}$ Surgery is contraindicated in a few patients with advanced age and multiple comorbidities. Non-surgical gallbladder drainage (GBD) can be performed via a percutaneous transhepatic (PT) or an endoscopic route with or without an endoscopic ultrasound (EUS).

Perihepatic ascites and coagulopathy are relative contraindications for PT-GBD. PT-GBD causes postoperative pain in approximately $12 \%$ of patients. ${ }^{5}$ Additionally, catheter obstruction, dislodgement and migration require multiple post-procedural interventions and long-term care. PT-GBD can be use- 
ful as either a bridge to surgery or occasionally is planned as destination therapy, although patients may report discomfort from external drainage tubes and reject this procedure owing to aesthetic reasons.

In the absence of EUS, endoscopic drainage of the GB has been attempted via a transpapillary approach aimed at stenting the cystic duct. This technique is feasible only if the cystic duct shows opacification on a cholangiogram and a guide-wire can be successfully passed into the cystic duct. Reportedly, the technical success rate (TSR) of this approach is approximately $91 \%{ }^{6}$ Common complications include bleeding, stent migration or occlusion, sedation-related adverse events, and those associated with the endoscopic retrograde cholangiopancreatography (ERCP) procedure itself.

EUS-GBD is a relatively newer application of the EUS-guided approach to create an iatrogenic fistula between the GB and the gastrointestinal (GI) tract. EUS-GBD has been performed using plastic stents, nasobiliary drainage tubes, or self-expandable metal stents (SEMS); however, the high risk of stent migration and bile leakage secondary to lack of apposition of the luminal walls has been considered an obstacle to achieving safe clinical outcomes. A lumen-apposing metal stent (LAMS) is a saddle-shaped stent designed from braided nitinol wires with double-walled flanges perpendicular to the lumen on both sides to provide adequate anchorage across the non-adherent luminal structures. It is completely covered with silicone to counteract tissue ingrowth and tract leakage and to allow removability. In this article, we summarize the indications, technique, outcomes, and adverse events associated with EUSGBD using LAMS based on our review of 10 original studies.

\section{MATERIALS AND METHODS}

Two authors individually reviewed the English literature from inception through September 2017. PubMed and Google Scholar were used to identify peer-reviewed original and review articles using the following key words: "Lumen-apposing metal stents (LAMS)," "gallbladder drainage", and "endoscopic ultrasound". Only studies involving humans were selected. The references cited for pertinent studies were manually searched to identify additional relevant studies. Our search results yielded 10 studies. ${ }^{7-16}$ Indications, procedural details, technical and clinical outcomes, as well as adverse events with their management were reviewed for each study.

\section{RESULTS}

Ten original studies were included in our review article.
All were retrospective studies ${ }^{7-15}$ except $1 .^{16}$ Three studies enrolled patients from multiple centers ${ }^{11,15,16}$ and the rest were single-center studies. ${ }^{7-10,12-14}$ The reported literature described studies from the US, ${ }^{8,10-13,15}$ Spain, Japan, ${ }^{9}$ Germany ${ }^{14}$ and the Netherlands. ${ }^{16}$ Details regarding all studies have been summarized in Table 1.

\section{Patient characteristics}

Most patients in our study cohort were aged $\geq 60$ years $^{7-16}$ with only a few patients aged $<50$ years. ${ }^{11,13,15}$ Our study showed an even distribution of women $(47.1 \%)$ and men $(52.9 \%)$ in a ratio of 1:1.1. ${ }^{7-16}$ The retrospective studies that compared outcomes between EUS-GBD and PT-GBD showed no statistically significant intergroup differences in terms of baseline population characteristics, etiology of cholecystitis, site of obstruction, median thickness of the GB wall, median duration of cholecystitis, and antibiotic exposure prior to the planned intervention. ${ }^{15}$

\section{Indications}

The most common indication for the procedure was acute cholecystitis ${ }^{7,911-16}$ in addition to acute cholangitis, ${ }^{8} \mathrm{~GB}$ hydrops ${ }^{10,11}$ or biliary obstruction. ${ }^{11}$ The underlying etiology was either an isolated condition or a combination of cholelithiasis, choledocholithiasis, acalculous cholecystitis, or malignancies such as pancreatic adenocarcinoma, cholangiocarcinoma, or GB adenocarcinoma. ${ }^{7-16}$ All such patients had been deemed poor surgical candidates and had either refused to undergo PT-GBD or preferred to undergo EUS-GBD. ${ }^{7-12,14-16}$ A study showed that all patients who underwent PT-GBD continued to remain poor surgical candidates during follow-up and required management with internal drainage using EUS-GBD. ${ }^{13}$ In patients with biliary obstruction, ERCP failed to relieve obstruction secondary to technically challenging anatomy. ${ }^{8}$ In addition to patient preference, PT-GBD was considered unsafe in a few patients with concomitant perihepatic ascites, or coagulopathy, and in those who needed to continue the use of blood thinners, or were at a high risk of tube dislodgement (patients with dementia, and agitated behavior, among other such conditions). ${ }^{11}$

\section{Procedural characteristics}

\section{Anesthesia}

Only 4 studies described the nature of anesthesia used for the procedure, which varied from general, ${ }^{11,15}$ and monitored anesthesia ${ }^{14,16}$ to conscious sedation. ${ }^{16}$ These results are highly center specific. No patient-reported outcomes are available to compare procedure tolerance across different studies, although the TSR remained comparable independent of the 


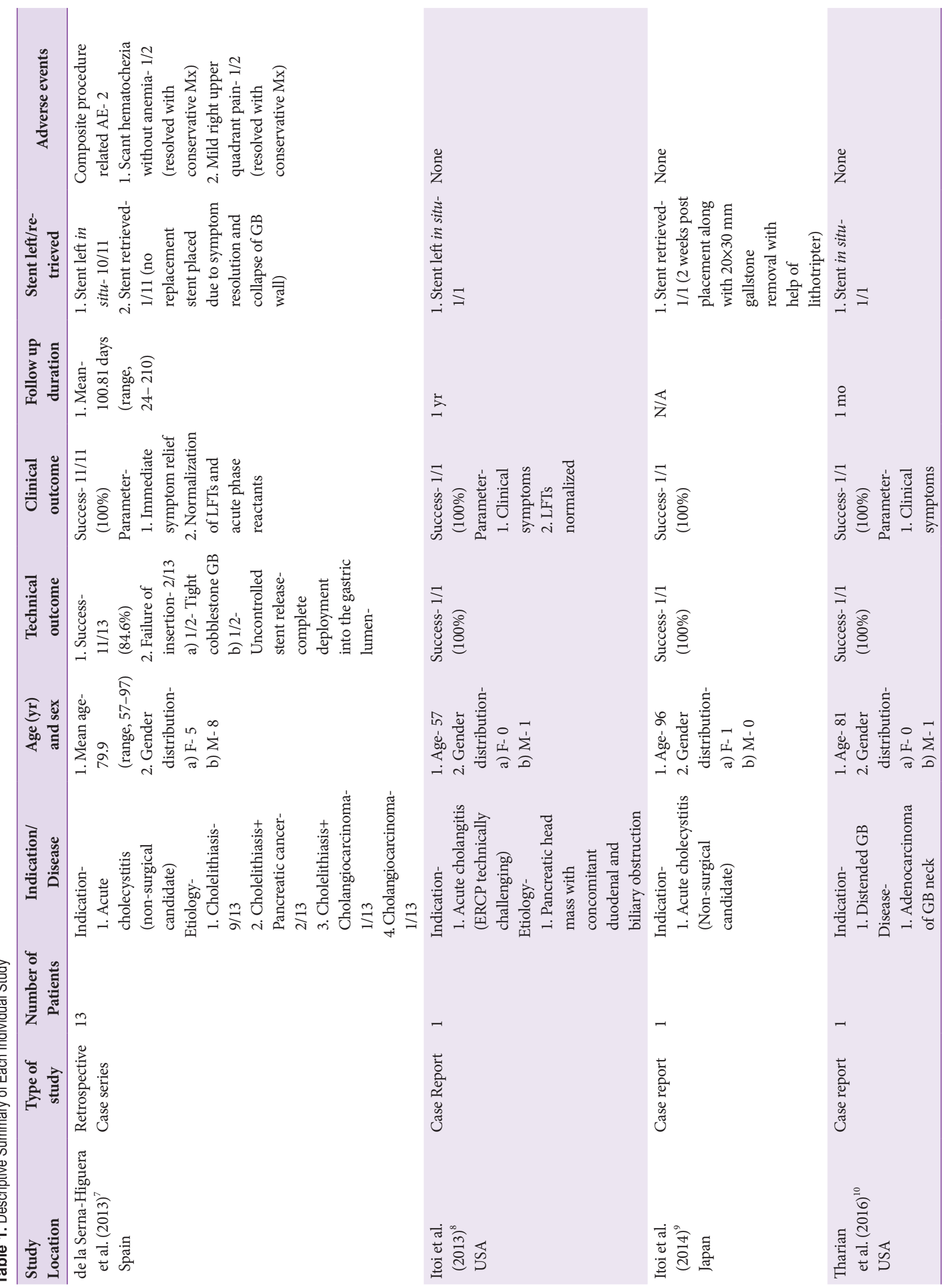




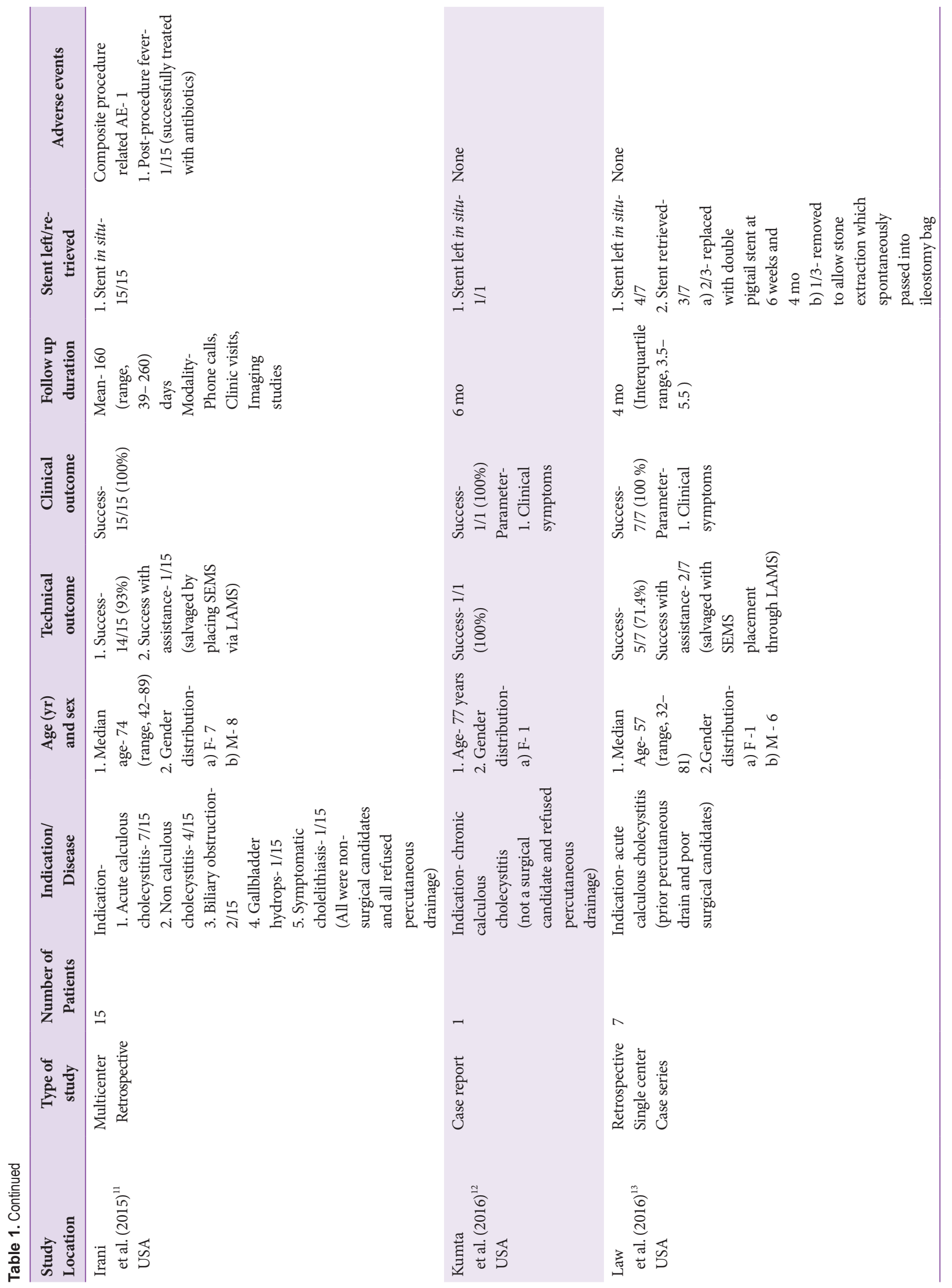


C clinical endoscopy

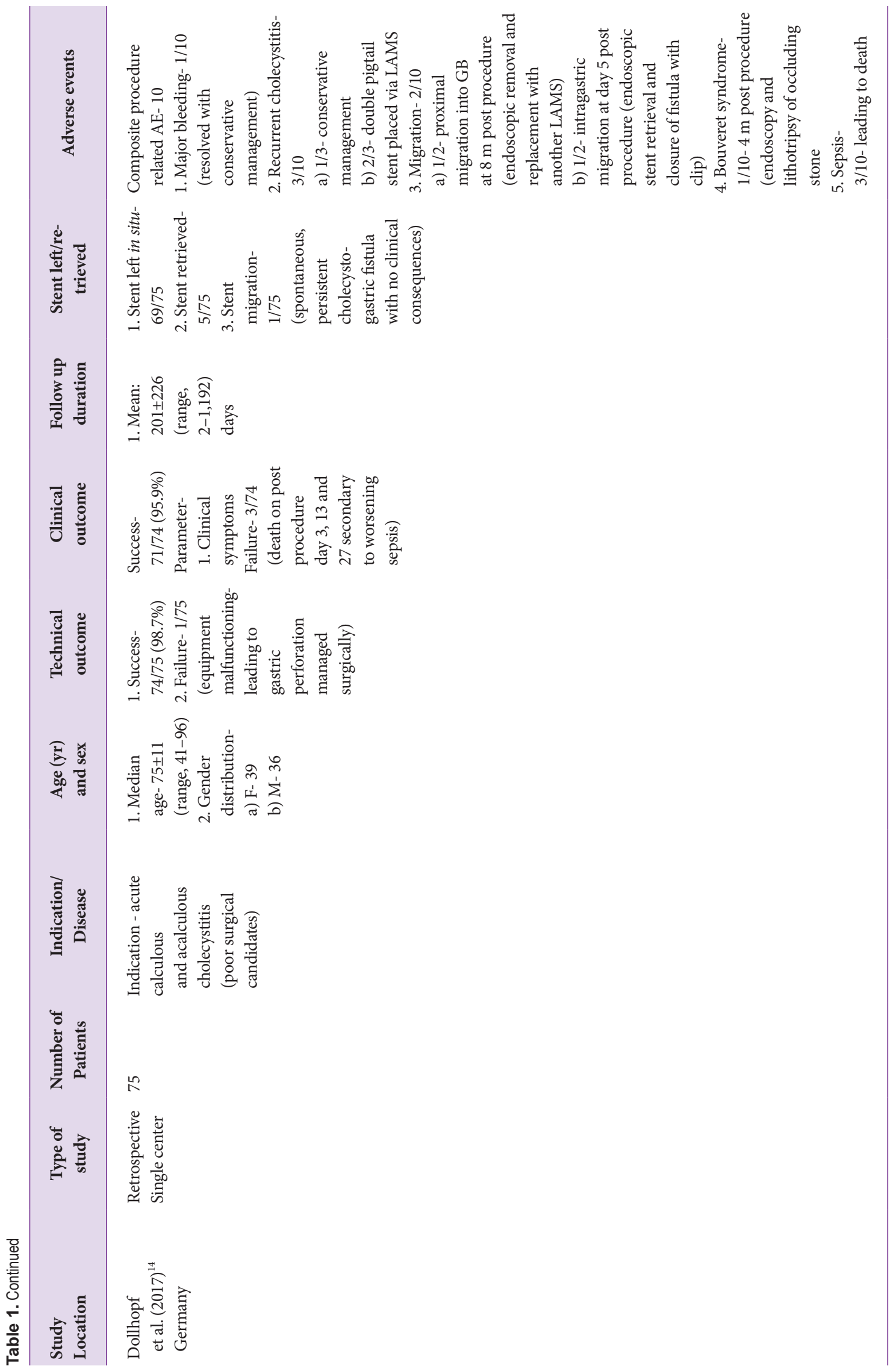




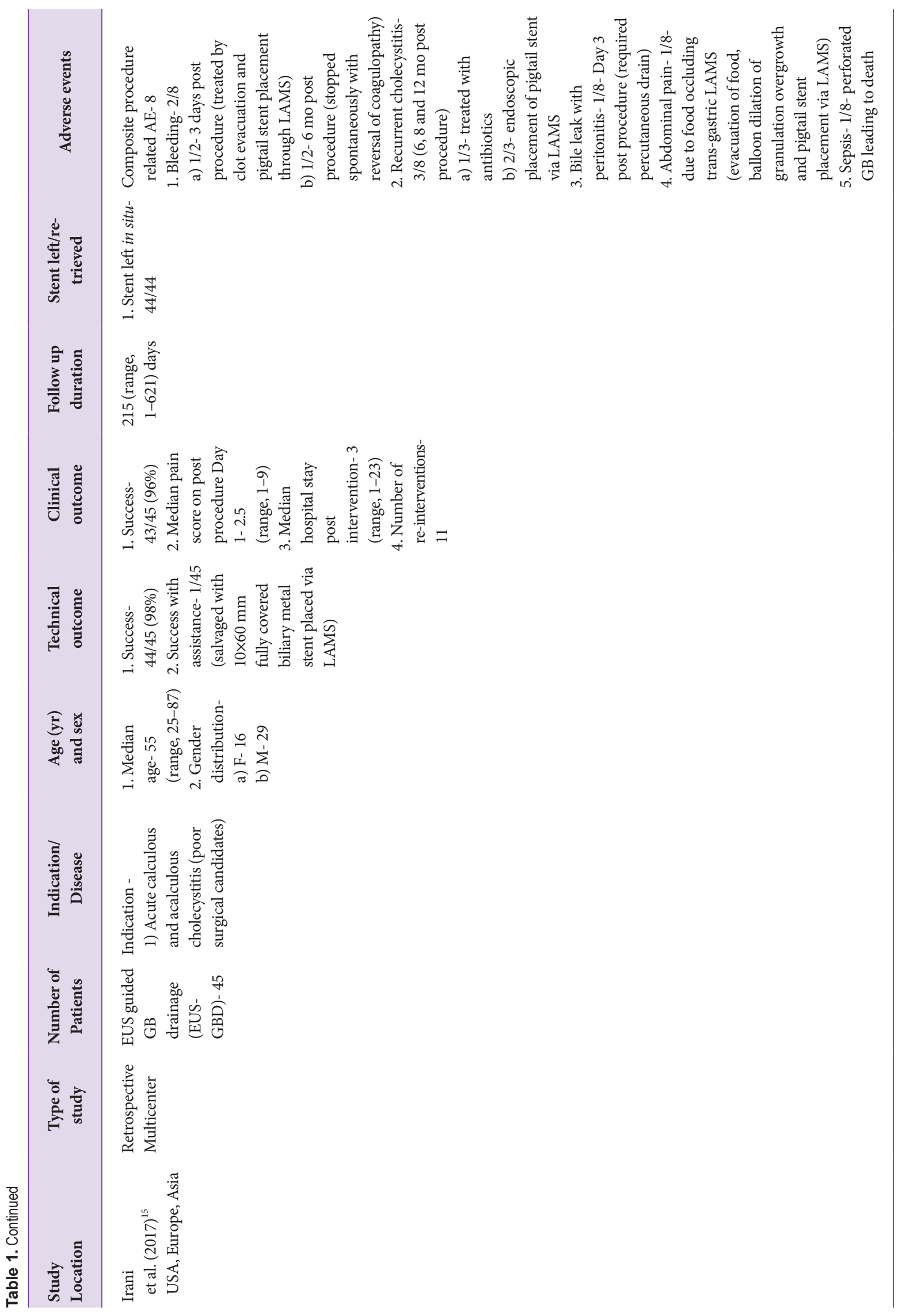


C clinical endoscopy

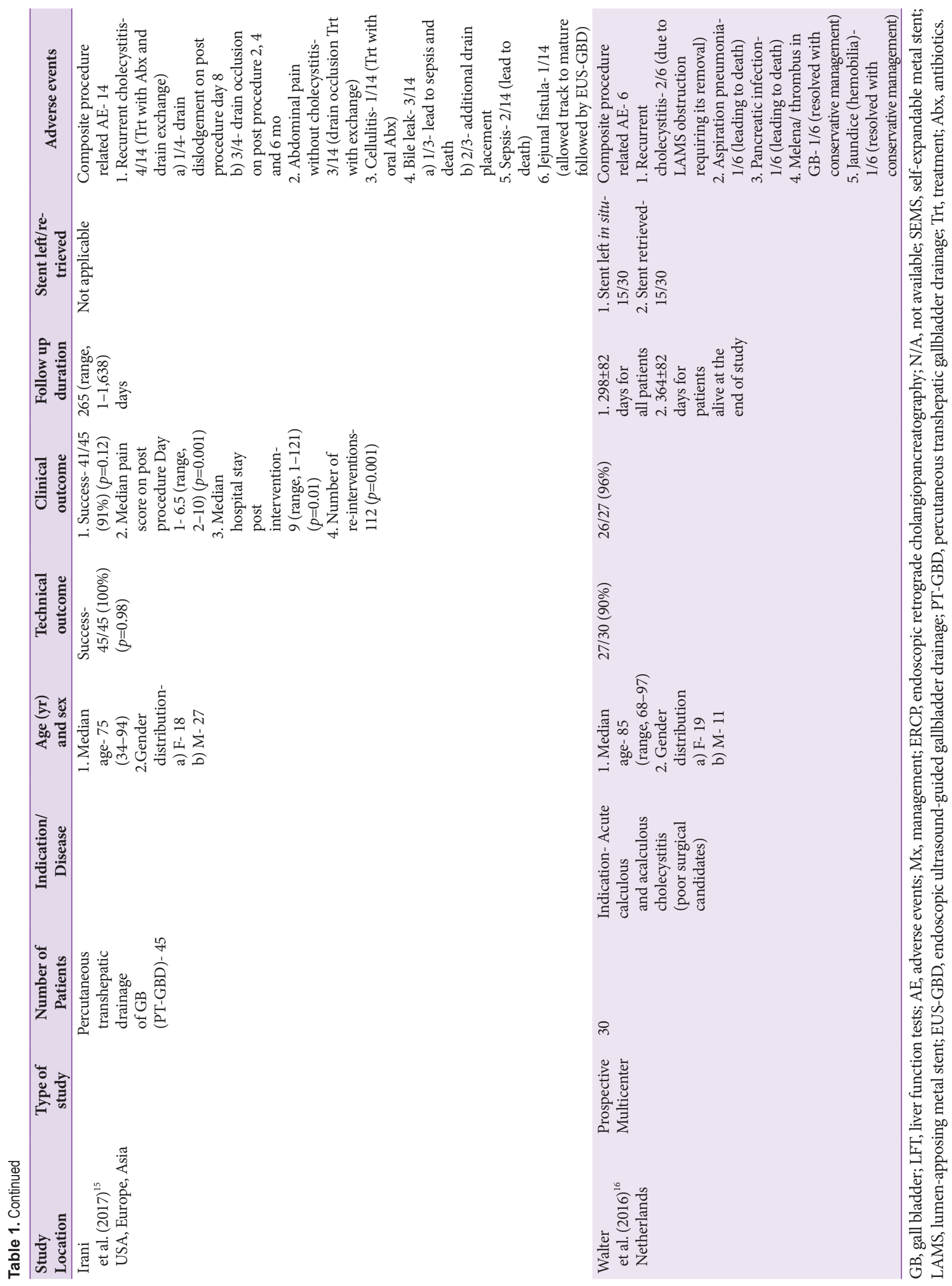


type of anesthesia used.

\section{Stent specifics}

The LAMS is designed to allow apposition of the luminal walls of 2 non-adherent organs. The endoscopist determines the size and length of the LAMS to be used after considering the patient-specific anatomy, GB wall thickness, and diameter of in situ GB stones (to allow unobstructed passage of the stent). Details regarding stents used by different studies have been summarized in Table 2. Because of increasing experience with the use of LAMS, a few authors recommend prophylactic placement of a SEMS or a plastic pigtail stent through the LAMS to prevent future occlusion and displacement. ${ }^{7,11,13,15}$ This practice is more useful in patients in whom the LAMS is intended to remain in situ indefinitely, to avoid the need for re-intervention. In addition to the above-mentioned studies, 2 authors used SEMS to provide additional length when the proximal flange of the LAMS was noted to deploy into the extraluminal space ${ }^{11,13,15}$ potentially salvaging a technical failure.

In our review, approximately $50 \%$ of the operations $(48.2 \%)$ were performed using cautery enhanced LAMS ${ }^{12,14,15}$ and the rest were performed using non-cautery enhanced LAMS $(51.8 \%)^{7-11,13,15,16}$

\section{Site of puncture}

Most studies did not explain their choice of a transgastric vs. a transduodenal or transjejunal approach. This decision is left to the discretion of the endoscopist after assessing the patient's anatomy to determine the site of maximal direct apposition between the GB wall and the GI tract to allow LAMS placement. LAMS placement in porcine models is known to cause mature fistula tract formation within $4-5$ weeks. ${ }^{17}$ The LAMS tends to cause significant tissue overgrowth, which serves as a drawback during stent removal. Walter et al. proposed that the retroperitoneal location of the duodenum allows stable tract formation compared to the stomach where peristaltic movements lead to a higher degree of tissue reaction consequently leading to tissue overgrowth. ${ }^{16}$

In our review comprising 189 patients, 75 (39.7\%) underwent transgastric, ${ }^{7-9,11,14-16} 113$ (59.8\%) underwent transduodenal, ${ }^{7,10-16}$ and $1(0.5 \%)$ patient underwent transjejunal puncture ${ }^{14}$ to access the GB.

\section{Technique}

All authors used an oblique/forward viewing therapeutic linear array echoendoscope to visualize the GB. A standard 19-gauge needle was used to puncture the gastric antrum or the duodenal bulb to access the GB wall, and entry into the GB was confirmed using real-time ultrasound and color Doppler imaging. Next, the contrast agent was injected to obtain fluoroscopic images of the biliary tree. A standard biliary guide-wire was coiled into the GB lumen. The tract was then dilated over the guide-wire using a balloon dilator or cystotome. A preloaded stent was then advanced over the guide-wire. The distal flange of the stent was first deployed into the GB and abutted against the wall under ultrasonographic or fluoroscopic guidance. Next, the proximal flange of the stent was deployed into the duodenum or the stomach under direct visualization, thereby performing cholecystoenterostomy or cholecystogastrostomy, respectively. The stent position was confirmed endoscopically or fluoroscopically and via aspiration of biliary contents. If a cautery enhanced LAMS is being used, the puncture site, dilation of the tract and stent deployment can all be performed simultaneously, thereby potentially decreasing the procedure time.

\section{Procedure time}

Only 4 studies reported procedure times, ${ }^{11,14-16}$ which varied widely between a minimum of $8 \mathrm{~min}^{14}$ and a maximum of 110 min. ${ }^{16}$ The mean procedure time varied from $22-38 \mathrm{~min} .11,14,15$ The mean EUS-GBD procedure time was 6 min longer than that of a PT-GBD $(p=0.02){ }^{15}$ Dollhopf et al. reported a significantly ( $p=0.04$ ) shorter stent deployment time with the use of cautery enhanced LAMS (3.1 $\mathrm{min}$ ) vs. non-cautery enhanced LAMS $(7.7 \mathrm{~min}) .{ }^{14}$

\section{Technical outcome}

The TSR was determined by calculating the percentage of patients who underwent successful LAMS deployment across the GB wall with drainage into the stomach or the small bowel. The composite TSR (without SEMS) for EUS-GBD was $95.2 \%(180 / 189))^{7-16}$ The LAMS could not initially be deployed in 3 patients; however, a SEMS was successfully placed through the LAMS to bridge the gap across the GB wall to overcome this difficulty. ${ }^{11,1,15}$ The composite TSR (with SEMS) for EUSGBD was $96.8 \%(183 / 189)^{7-16}$ The individual TSR (without SEMS) varied between $71.4 \%^{13}$ and $100 \% \%^{8-10,12}$ The individual TSR (with SEMS) varied between $84.6 \%^{7}$ and $100 \%{ }^{8-13,15}$ Reasons for failure $(n=6)$ could be attributed to a stiff cobblestone GB (1/6), uncontrolled stent release (1/6), equipment malfunction leading to gastric perforation $(1 / 6),{ }^{14}$ and unknown etiology $(3 / 6){ }^{16}$ A study performed by Irani et al. showed that the TSR was comparable between EUS-GBD and PT-GBD $(p=0.98) .{ }^{15}$

\section{Clinical outcomes}

The clinical success rate (CSR) was measured by calculating the percentage of patients who showed significant improvement in clinical, laboratory, or radiological parameters post EUS-GBD via LAMS (with or without SEMS). The composite CSR was $96.7 \%(177 / 183){ }^{7-16}$ The individual CSR varied be- 
C clinical endoscopy

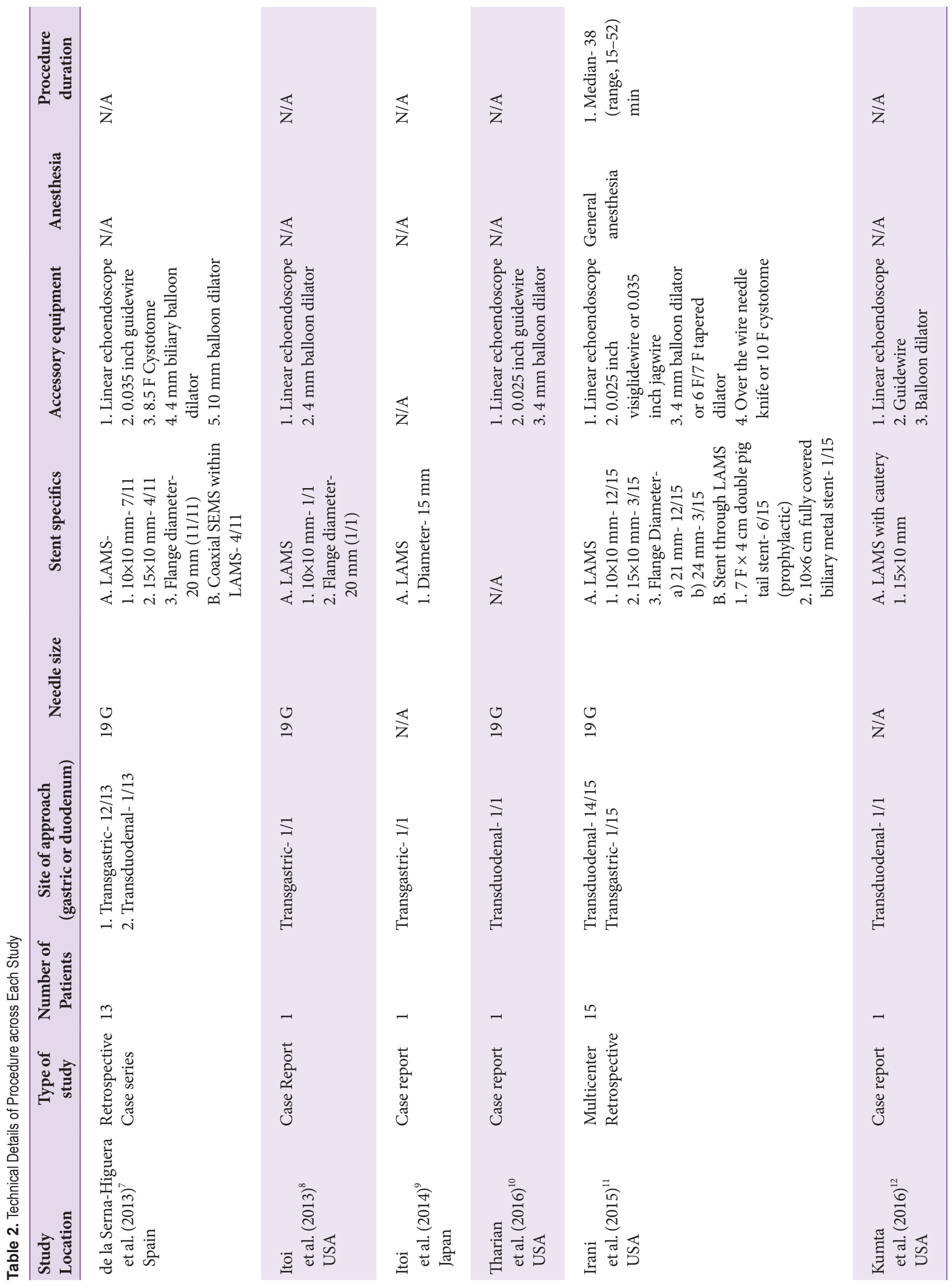




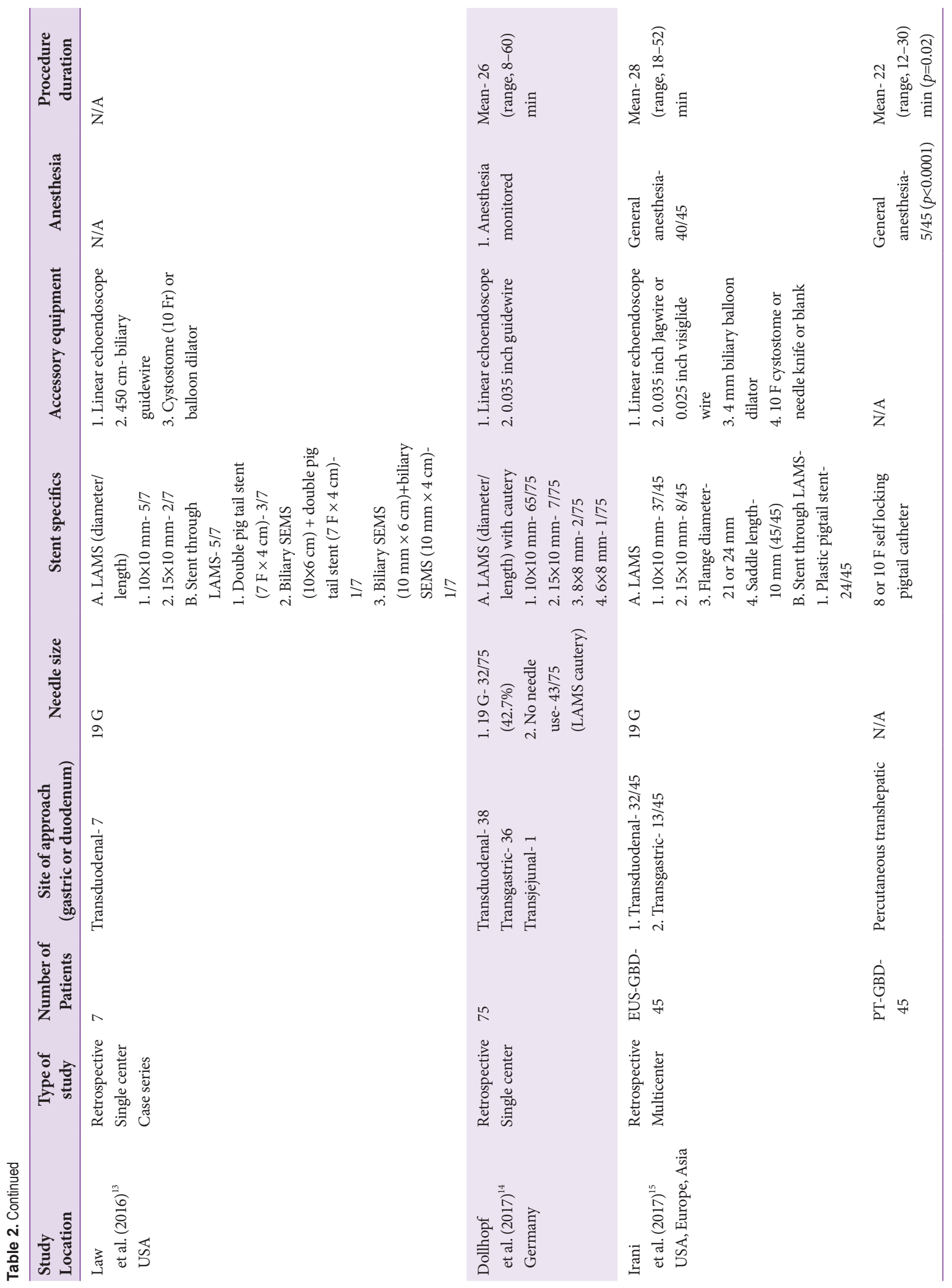




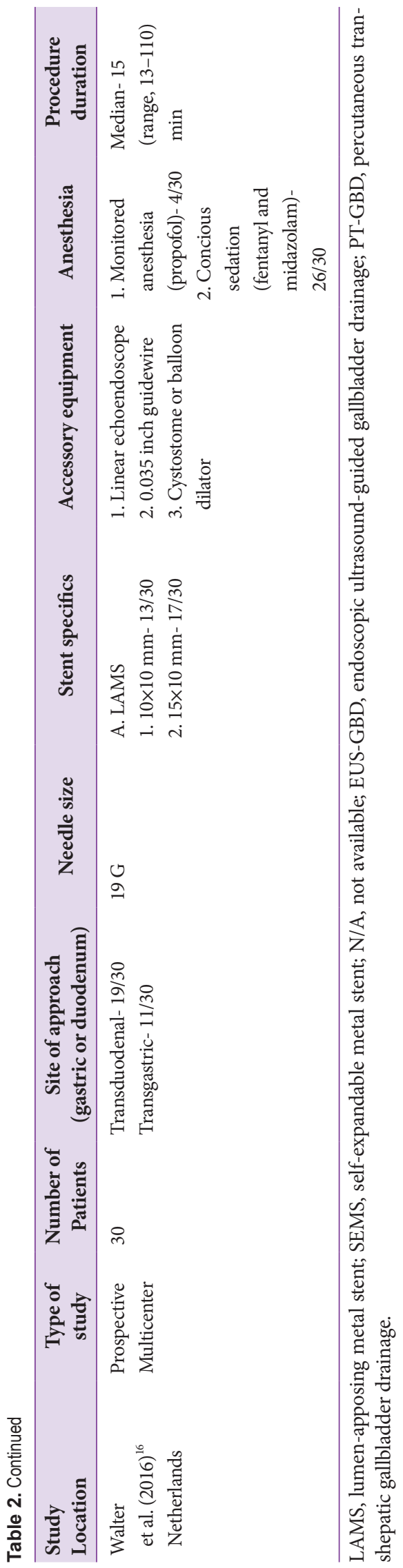

tween $95.9 \%{ }^{14}$ and $100 \%{ }^{7-13}$ Among the 6 patients who showed a lack of response, GB wall perforation was considered the possible etiology in $2{ }^{14,15}$ and no obvious reason could be detected in the remaining 4 patients other than coexistent comorbidities. ${ }^{14-16}$ The CSR of EUS-GBD (96\%) was higher than that of PT-GBD (91\%), although this result was not statistically significant $(p=0.12) .{ }^{15}$ Notably, the authors demonstrated a statistically significant decrease in the postoperative pain on day $1(p=0.001)$, a shorter median length of hospitalization post-intervention ( $p=0.01)$, as well as fewer re-interventions $(p=0.001)$ with EUS-GBD than with PT-GBD. ${ }^{15}$

\section{Adverse events}

Sepsis

Lack of resolution of acute cholecystitis despite stent placement resulted in progression to sepsis, necrosis of the GB wall, perforation, and death. ${ }^{14,15,16}$ These complications have been presented in detail under Clinical Outcomes.

\section{Recurrent cholecystitis}

Among the 177 patients who showed initial clinical resolution of symptoms, 9 (5.1\%) presented with a repeat attack of acute cholecystitis. This condition usually occurs secondary to either stent dislodgement (usually in the early post-procedural period before the fistula matures into a tract), or occlusion (an early or late post-procedural complication) secondary to gallstones, food bolus, clots, or tissue overgrowth over time. Patients may improve with conservative management (antibiotics) alone ${ }^{14,15}$ or may require endoscopic debridement with placement of a pigtail stent through the LAMS or complete removal of the LAMS. A few authors recommend prophylactic placement of pigtail stents through the LAMS at the time of initial procedure to prevent this complication. ${ }^{7,11,13,15}$ A few authors also recommend performing endoscopic lavage with/ without stone extraction via the LAMS after placement to prevent future attacks. ${ }^{7}$

\section{Bleeding}

Intraprocedural bleeding is an expected outcome because the procedure involves the creation of a transmural fistula between the GI tract and the GB wall. Bleeding was easily controlled in most patients and was not reported as a complication. Among 189 patients, 5 (2.6\%) reported post-procedural GI bleeding, ${ }^{7-16}$ which in 4/5 patients were managed with conservative treatment alone (reversal of coagulopathy, administration of intravenous fluids, and other such interventions)., ${ }^{7,14-16}$ In 1 patient, endoscopic evaluation revealed a clot without active bleeding, which was treated with clot evacuation and placement of a pigtail stent through the LAMS. ${ }^{15}$ One patient 
developed hemobilia, which improved with supportive care alone. $^{16}$

\section{Stent migration}

Stent migration is a relatively rare complication with LAMS placement compared to the placement of SEMS or pigtail catheters.

Among the 183 patients who underwent EUS-GBD via LAMS, only $2(1.1 \%)$ showed stent migration. ${ }^{7-16}$ One event occurred on post-procedure day 5 (managed with stent retrieval and clip closure of the fistula) and the other occurred 8 months post procedure (managed with LAMS replacement). ${ }^{14}$

\section{Miscellaneous}

These complications noted in the composite study cohort $(n=189)$ included abdominal pain in $1.1 \%$ of patients (1 patient developed stent occlusion from a food bolus requiring endoscopic debridement and pigtail stent placement, and the other improved with supportive care alone) ${ }^{7,15}$ fever in $0.5 \%$ (improved with supportive care), ${ }^{11}$ bile leak with peritonitis in $0.5 \%$ (managed with percutaneous drain placement), ${ }^{15} \mathrm{Bou}$ veret syndrome in $0.5 \%$ (treated with endoscopic lithotripsy and stone removal), ${ }^{14}$ aspiration pneumonia in $0.5 \%$ (eventually leading to death) ${ }^{16}$ and pancreatic infection in $0.5 \%$ (eventually leading to death). ${ }^{16}$

\section{CONCLUSIONS}

The absence of an external drainage tube and widespread applicability in patients with coagulopathy or ascites make EUS-GBD using LAMS an attractive option for patients with acute cholecystitis in whom surgery is contraindicated. The procedure demonstrates a high TSR and CSR, although this evidence is based on the few high-volume centers where only a small number of experts perform this procedure. A learning curve has been suggested; however, the minimum training requirements are yet to be determined. Complications although rare, are associated with the risk of worsening the clinical situation in already sick patients who are poor surgical candidates. Based on the available evidence, EUS-GBD using LAMS is a safe and efficacious alternative when performed by experts in high-risk surgical patients with acute cholecystitis.

\section{Conflicts of Interest}

The authors have no financial conflicts of interest.

\section{Author Contributions \\ Conceptualization: Deepanshu Jain}

Data curation: DJ, Bharat Singh Bhandari, Nikhil Agrawal

Formal analysis: DJ

Investigation: DJ, Shashideep Singhal

Methodology: DJ

Project administration: DJ, SS

Resources: DJ

Supervision: DJ, SS

Validation: DJ, SS

Visualization: DJ

Writing-original draft: DJ, BSB, NA

Writing-review\&editing: DJ, SS

\section{REFERENCES}

1. Hassler KR, Jones MW. Gallbladder, cholecystectomy, laparoscopic [Internet]. Treasure Island (FL): StatPearls Publishing; c2017 [updated 2017 Oct 16; cited 2018 Apr 12]. Available from: https://www.ncbi.nlm.nih. gov/books/NBK448145/.

2. Friedman GD, Raviola CA, Fireman B. Prognosis of gallstones with mild or no symptoms: 25 years of follow-up in a health maintenance organization. J Clin Epidemiol 1989;42:127-136.

3. Garden OJ, Bradbury AW, Forsythe JLR. Principles and practice of surgery. 4th ed. Edinburgh: Churchill Livingstone; 2002.

4. Zacks SL, Sandler RS, Rutledge R, Brown RS Jr. A population-based cohort study comparing laparoscopic cholecystectomy and open cholecystectomy. Am J Gastroenterol 2002;97:334-340.

5. Itoi T, Sofuni A, Itokawa F, et al. Endoscopic transpapillary gallbladder drainage in patients with acute cholecystitis in whom percutaneous transhepatic approach is contraindicated or anatomically impossible (with video). Gastrointest Endosc 2008;68:455-460.

6. Widmer J, Alvarez P, Sharaiha RZ, et al. Endoscopic gallbladder drainage for acute cholecystitis. Clin Endosc 2015;48:411-420.

7. de la Serna-Higuera C, Pérez-Miranda M, Gil-Simón P, et al. EUS-guided transenteric gallbladder drainage with a new fistula-forming, lumen-apposing metal stent. Gastrointest Endosc 2013;77:303-308.

8. Itoi T, Binmoeller K, Itokawa F, Umeda J, Tanaka R. Endoscopic ultrasonography-guided cholecystogastrostomy using a lumen-apposing metal stent as an alternative to extrahepatic bile duct drainage in pancreatic cancer with duodenal invasion. Dig Endosc 2013;25 Suppl 2:137-141.

9. Itoi T, Itokawa F, Tsuchiya T, Kurihara T, Tanaka R. Transgastric large gallstone extraction through a lumen-apposing metal stent in a patient with acute cholecystitis. Gastrointest Endosc 2014;79:547.

10. Tharian B, Varadarajulu S, Hawes R. Drainage of obstructed gallbladder with use of lumen-apposing metal stent. Gastrointest Endosc 2016;83:460461.

11. Irani S, Baron TH, Grimm IS, Khashab MA. EUS-guided gallbladder drainage with a lumen-apposing metal stent (with video). Gastrointest Endosc 2015;82:1110-1115.

12. Kumta NA, Lordello Passos M, Rodela Silva GL, Novikov A, Kahaleh M. Endoscopic ultrasound-guided transmural gallbladder drainage with a lumen-apposing metal stent using an electrocautery enhanced delivery system. Endoscopy 2016;48:E327.

13. Law R, Grimm IS, Stavas JM, Baron TH. Conversion of percutaneous cholecystostomy to internal transmural gallbladder drainage using an endoscopic ultrasound-guided, lumen-apposing metal stent. Clin Gastroenterol Hepatol 2016;14:476-480.

14. Dollhopf M, Larghi A, Will U, et al. EUS-guided gallbladder drainage in patients with acute cholecystitis and high surgical risk using an electrocautery-enhanced lumen-apposing metal stent device. Gastrointest Endosc 2017;86:636-643.

15. Irani S, Ngamruengphong S, Teoh A, et al. Similar efficacies of endoscopic ultrasound gallbladder drainage with a lumen-apposing metal stent versus percutaneous transhepatic gallbladder drainage for acute cholecystitis. Clin Gastroenterol Hepatol 2017;15:738-745. 


\section{Ce cuncan enooscopy}

16. Walter D, Teoh AY, Itoi T, et al. EUS-guided gall bladder drainage with a lumen-apposing metal stent: a prospective long-term evaluation. Gut 2016;65:6-8
17. Binmoeller KF, Shah J. A novel lumen-apposing stent for transluminal drainage of nonadherent extraintestinal fluid collections. Endoscopy 2011;43:337-342. 